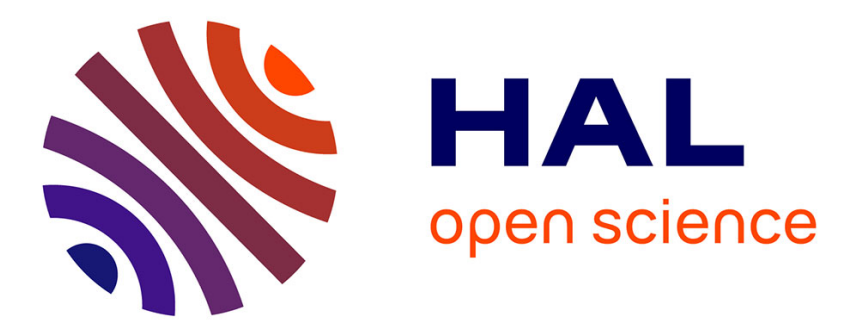

\title{
A premartensitic phase in KAlF4: neutron and X-ray scattering evidences
}

\author{
Alain Gibaud, Alain Bulou, A. Le Bail, J. Nouet, C.M.E. Zeyen
}

\section{To cite this version:}

Alain Gibaud, Alain Bulou, A. Le Bail, J. Nouet, C.M.E. Zeyen. A premartensitic phase in KAlF4: neutron and X-ray scattering evidences. Journal de Physique, 1987, 48 (9), pp.1521-1532. 10.1051/jphys:019870048090152100 . jpa-00210584

\section{HAL Id: jpa-00210584 https://hal.science/jpa-00210584}

Submitted on 1 Jan 1987

HAL is a multi-disciplinary open access archive for the deposit and dissemination of scientific research documents, whether they are published or not. The documents may come from teaching and research institutions in France or abroad, or from public or private research centers.
L'archive ouverte pluridisciplinaire HAL, est destinée au dépôt et à la diffusion de documents scientifiques de niveau recherche, publiés ou non, émanant des établissements d'enseignement et de recherche français ou étrangers, des laboratoires publics ou privés. 
Classification

Physics Abstracts

$61.50 \mathrm{~K}-61.70 \mathrm{~L}-64.70 \mathrm{~K}$

\title{
A premartensitic phase in $\mathrm{KAlF}_{4}$ : neutron and X-ray scattering evidences
}

\author{
A. Gibaud, A. Bulou, A. Le Bail (*), J. Nouet and C. M. E. Zeyen ( $\left.{ }^{+}\right)$ \\ Laboratoire de Physique de l'Etat Condensé, Université du Maine, \\ U.A. $n^{\circ} 807$ C.N.R.S., route de Laval, 72017 Le Mans Cedex, France \\ (*) Laboratoire des Oxyfluorures Ioniques, Université du Maine, \\ U.A. $n^{\circ} 449$ C.N.R.S., route de Laval, 72017 Le Mans Cedex, France \\ $\left(^{+}\right)$Institut Laue Langevin, $156 \mathrm{X}$ Centre de Tri, 38042 Grenoble Cedex, France
}

(Reçu le 2 mars 1987, accepté le 13 mai 1987)

\begin{abstract}
Résumé. - Nous montrons par diffraction de neutrons sur monocristal que les anomalies récemment observées dans les diagrammes de diffraction de rayons $\mathrm{X}$ dans la phase haute température de $\mathrm{KAlF}_{4}$ résultent de l'existence de domaines embryonnaires d'une phase prémartensitique coexistant de façon cohérente avec la phase haute température. L'arrangement des domaines ainsi que les paramètres de maille de la phase prémartensitique sont déterminés. En nous appuyant sur le caractère bidimensionnel de ce composé à structure en couches et sur des considérations géométriques, nous proposons une structure pour la phase prémartensitique. Nous interprétons la coexistence des deux phases par la présence de dislocations coins qui se forment vraisemblablement au cours de la croissance du matériau. Le rôle joué par les domaines prémartensitiques dans la transformation martensitique de $\mathrm{KAlF}_{4}$ est discuté.
\end{abstract}

\begin{abstract}
From elastic neutron scattering experiments, it is shown that the anomalies which have been recently observed in the high temperature phase of $\mathrm{KAlF}_{4}$ by the $\mathrm{X}$-ray mono-Laue technique can be attributed to embryonic domains of a premartensitic phase which coherently coexists with the high temperature phase. The arrangement of the domains and the lattice parameters of the premartensitic phase are determined. On the basis of geometrical considerations and with regard to the two-dimensional character of this layered compound we propose a possible structure for the premartensitic phase. The coexistence of the two phases can be interpreted by means of edge dislocations which are presumably formed during the crystal growth procedure. The role played by the premartensitic phase on the onset of the $\mathrm{KAlF}_{4}$ martensitic transformation is discussed.
\end{abstract}

\section{Introduction.}

The $\mathrm{ABX}_{4}$ layered compounds which derive either from the ideal $\mathrm{TlAlF}_{4}$ structure or from the ideal $\mathrm{KFeF}_{4}$ structure are presently the object of numerous studies owing to the variety of the structural phase transitions they exhibit. In this paper we are interested in $\mathrm{KAlF}_{4}$ which undergoes a SPT from the former structure to the latter one. In the previous investigations of this compound the high and low temperature phases have been determined [1-6]. It was shown [3] that the first order transition which occurs at $T_{\mathrm{c}}=260 \mathrm{~K}$ transforms the $\mathrm{KAlF}_{4}$ parent phase with the $D_{4 h}^{5}-P 4 / m b m$ symmetry into a product one with the $\mathrm{C}_{2 \mathrm{~h}}^{2}-\mathrm{P} 2 / \mathrm{m}$ symmetry according to a reconstructive mechanism. This mechanism is consistent with a martensitic transformation and in this paper it is shown that $\mathrm{KAlF}_{4}$ can be considered as one of the rare ionic compounds undergoing this sort of transformation which is usually observed in metallic alloys $(*)$. Among these features there are the diffraction anomalies which have been recently observed by the X-ray mono-Lauë technique and which might be regarded as premonitory effects of the martensitic transformation [5]. These premartensitic (PM) effects consist of unexpected extra-spots located nearly at the exact place where the most intense Bragg reflections of the product phase grow

(*) A detailed review of martensitic transformations occurring in inorganic crystals, minerals and ceramics is given in reference [7]. Generalities concerning the martensitic transformation can be obtained for example in references [8] and [9]. 
whilst the parent phase reflections remain unaltered. Such effects have already been observed in metallic alloys but the explanation of their origin and their connection with the martensitic phase has given rise to controversy [10-13].

Thus, the aim of this present study is to investigate the nature of the PM effects by a neutron four-circle diffraction experiment. The existence of extra-spots is discussed in the context of a phase coexistence which precludes a description in terms of an incommensurate phase and a dislocation-mediated mechanism is proposed to explain the origin of the phase coexistence.

\section{On the martensitic transformation in $\mathbf{K A l F}_{4}$.}

The recent investigations of $\mathrm{KAlF}_{4}$ have revealed that this compound undergoes at $T_{\mathrm{c}}=260 \mathrm{~K}$ a sudden phase transition which presents many characteristic features of a martensitic transformation. The transition has been shown to proceed from a shuffledominated mechanism which seems to be driven by the softening of a phonon branch $[2,6]$. To understand the reconstructive mechanism, let us first describe the high and low temperature phases of $\mathrm{KAlF}_{4}$ :

- the high temperature phase (also denoted phase II) derives from the ideal $\mathrm{TlAlF}_{4}$ tetragonal structure (also denoted phase I) by octahedra rotations around the [001] axis as shown in figure 1a. Thus, this structure is made up of two-dimensional arrays of $\mathrm{AlF}_{6}^{3-}$ octahedra as illustrated in figure $1 \mathrm{~b}$. The rotation of octahedra produces the doubling of the ideal unit cell in the basal plane so that the space group is - $D_{4 h}^{5} P 4 / m b m-\left(\right.$ instead of $D_{4 h}^{1} P 4 / m m m$ for the ideal phase) with lattice parameters given in table I. The rotation angle is roughly equal to $11^{\circ}$. (Lattice vectors in phases I and II are related as follows : $\mathbf{a}_{\mathrm{II}}=\mathbf{a}_{\mathrm{I}}+\mathbf{b}_{\mathrm{I}} \mathbf{b}_{\mathrm{II}}=-\mathbf{a}_{\mathrm{I}}+\mathbf{b}_{\mathrm{I}} \mathbf{c}_{\mathrm{II}}=\mathbf{c}_{\mathrm{I}}$ );

- the low temperature phase (also denoted phase III) is composed of staggered adjacent $\mathrm{AlF}_{6}^{3-}$ octahedra which are no more on the top of each other as it was the case in phase II (Fig. 1c). Thus phase III structure is very similar to the ideal $\mathrm{KFeF}_{4}$ structure (Fig. 1d) which belongs to the $\mathrm{Bmmb}$ space group. However in $\mathrm{KAlF}_{4}$ the $\mathrm{AlF}_{6}^{3-}$ octahedra are rotated around the [001] axes so that the symmetry is $\mathrm{P} 2_{1} / \mathrm{m}$ with the lattice parameters given in table I. Nevertheless, as the monoclinic distortion is very small ( $\beta=90.18^{\circ}$ in the pseudoorthorhombic cell) it is convenient for simplicity to refer to the orthorhombic $\mathrm{KFeF}_{4}$ ideal structure.

The above structural description evidences that the SPT which occurs in $\mathrm{KAlF}_{4}$ proceeds from a shear transformation which results from the gliding of $\mathrm{AlF}_{6}^{3-}$ octahedra sheets in the $[100]_{\mathrm{I}}$ direction. The gliding is equal to half the $a_{\mathrm{I}}$ lattice parameter of

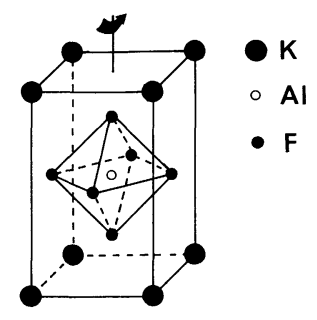

a)

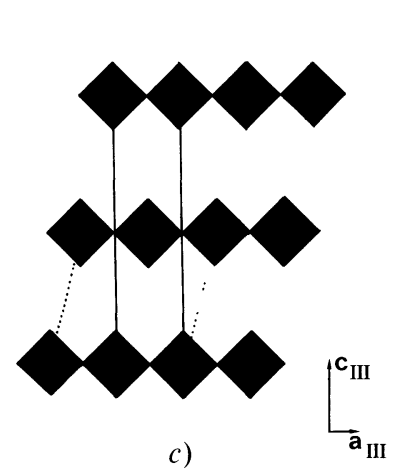

c)

Fig. 1. - Schematic representations of the $\mathrm{KAlF}_{4}$ structures in the parent and product phases : a) ideal $\mathrm{TlAlF}_{4}$ structure from which the $\mathrm{KAlF}_{4}$ parent phase is derived by the octahedra tilts indicated by the arrow, b) two-dimensional arrays of $\mathrm{AlF}_{6}$ octahedra in the parent phase, c) illustration of the staggered layers of octahedra in the product phase of $\mathrm{KAlF}_{4}$. The pseudo-orthorhombic cell is presented in full lines and the monoclinic one in doted lines, d) pseudo-orthorhombic $\mathrm{KAlF}_{4}$ structure in which the tilts which are observed in the product phase are arrowed (owing to the weak monoclinic distortion $\left(\beta=90.18^{\circ}\right)$ this structure is similar to the ideal orthorhombic $\mathrm{KFeF}_{4}$ structure).

Table I. - Lattice parameters of the parent and product phases of $\mathrm{KAlF}_{4}$ (Refs. [3, 4]). The product phase lattice parameters are given both for the pseudo-orthorhombic and monoclinic cells.

\begin{tabular}{c|c|c|c} 
& Phase II & \multicolumn{2}{|c}{ Phase III } \\
\hline \multirow{2}{*}{$\begin{array}{c}\text { Space } \\
\text { group } \\
\text { cell }\end{array}$} & $\mathrm{D}_{4 \mathrm{~h}}^{5}-\mathrm{P} 4 / \mathrm{mbm}$ & \multicolumn{2}{|c}{$\mathrm{C}_{2 \mathrm{~h}}^{2}-\mathrm{P} 2 / \mathrm{m}$} \\
\cline { 2 - 4 } & $\begin{array}{c}\text { tetragonal } \\
(4)\end{array}$ & $\begin{array}{c}\text { monoclinic } \\
(3)\end{array}$ & $\begin{array}{c}\text { pseudo- } \\
\text { orthorhombic } \\
(3)\end{array}$ \\
\hline$a(\AA)$ & $5.0431(1)$ & $7.3403(2)$ & 3.670 \\
$b(\AA)$ & $5.0431(1)$ & $7.2370(2)$ & 7.237 \\
$c(\AA)$ & $6.1567(1)$ & $6.4070(2)$ & 12.267 \\
$\beta$ & - & $106.801^{\circ}(4)$ & $90.180^{\circ}$
\end{tabular}

the ideal phase. Such a transition, characteristic of the layered arrangement, is similar to the martensitic transformation usually observed in metallic alloys and sometimes in some ionic compounds such as zirconia $\mathrm{ZrO}_{2}$ [14]. According to the terminology 
used in martensitic transformations, the high and low temperature phases will be respectively denoted the parent and product phases.

In addition to the structural evidences for a martensitic transformation, macroscopic effects are also observed. Indeed $\mathrm{KAlF}_{4}$ crystals suddenly break at the transition with a click-like nature which generally causes them to jump out of the support as observed in $\mathrm{NH}_{4} \mathrm{Br}$ [15]. Crystals become optically opaque and the surface of samples exhibit random cross-like cracks [3] which arise from the conflicting shape change of independent crystallites. Such effects have been recently analysed by the X-ray mono-Lauë technique [5]. Assuming that the gliding of $\mathrm{AlF}_{6}^{3-}$ octahedra sheets can occur with equal probabilities along the two $[100]_{\mathrm{I}}$ and $[010]_{\mathrm{I}}$ directions of the ideal tetragonal phase, transformed crystals are expected to be mainly formed of 6 variants (or domains) as illustrated in figure 2 and justified in the following. The simulation of the mono-Lauë pattern in the product phase has allowed the determination of the mean orientation of the variants. With respect to the pseudo-orthorhombic unit cell, two variants have their axes parallel to those of the ideal phase and four variants arising from crystallites which have glided in opposite directions are found to be mainly misoriented from $17^{\circ}$ around the $[010]_{\text {III }}$ or $[100]_{\text {III }}$ directions with a $\pm 5^{\circ}$ scatter about these new orientation directions ; note that misorientations as large as $25^{\circ}$ can yet be present in transformed crystals.

The kinetics of transformation is extremely quick and it is impossible even with an energetic quenching to avoid the transformation. The observation under

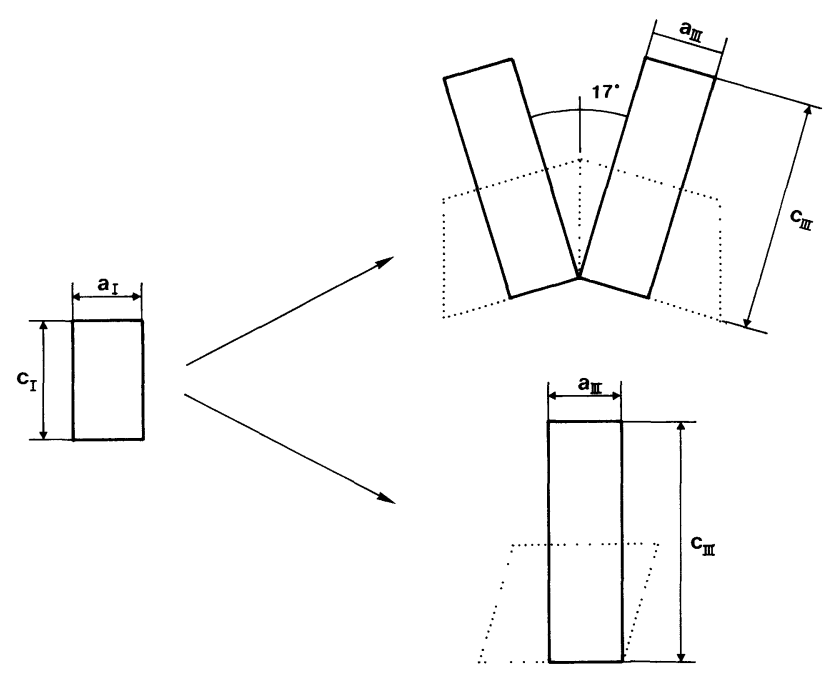

Fig. 2. - Illustration of the variants arrangement observed in the product phase of $\mathrm{KAlF}_{4}$ with respect to the pseudo-orthorhombic unit cell (in heavy lines). Only three variants are represented since the three others can be deduced from these ones by a rotation of $90^{\circ}$ around the $\left\langle c_{\mathrm{I}} »\right.$ axis. The monoclinic unit cell is shown in doted lines. microscope of the sudden and random appearence of cracks strongly suggests that the transformation is dominated by the nucleation rate and is not controlled by the growth rate. This point is confirmed by the fact that the intensity of the product phase Bragg reflections (in a fully transformed crystal) does not evolve below $T_{\mathrm{c}}$ on a subsequent cooling.

Moreover, similarly to what happens in the martensitic transformation of the $\mathrm{Fe}-\mathrm{Ni}$ alloy [16], a large hysteresis of $100{ }^{\circ} \mathrm{C}$ during which both phases coexist is observed. This suggests that the parentproduct phases interface does not move backwards on heating and is presumably pinned by its environment. Above $T_{\mathrm{c}}+100{ }^{\circ} \mathrm{C}$ the crystal is back into the parent phase but presents irreversible misorientations similar to those observed in the product phase which means that on heating the parent phase is nucleated within the immobilized product plates. The $\mathrm{KAlF}_{4}$ martensitic transformation can thus be identified to a non-thermoelastic transformation.

Additional information for a martensitic-like transformation in $\mathrm{KAlF}_{4}$ is the presence of extraspots in the X-ray mono-Lauë pattern of the parent phase [5]. As shown in figure 3 these spots lie along the [110] and [1̄o] directions of the parent phase. Some of them are superimposed on the diffuse scattering (DS) coming from the soft optic MX branch of phonons located between the 211 and 121 Bragg reflections but it must be pointed out that they do not arise from the condensation of a soft mode along this line since :

- no modification of the DS anisotropy is observed at these latter positions when $T_{\mathrm{c}}$ is closely approached from above ;

- the neutron investigation [2,6] of the phonon frequencies has not probed any «minimum minimorum » along the flat MX branch.

This statement is enhanced by the fact that in the mono-Lauë geometry the most intense extra-spots are found around the 201 Bragg reflection of the parent phase which is not surrounded by DS.

At $T_{c}$, extra-spots suddenly transform into the most intense Bragg reflections of the product phase. This strongly suggests that extra-spots could be considered as premonitory effects of the martensitic transformation and might arise from the existence of product phase domains in the parent phase though it is rather surprising when one thinks about the cooperative movements occurring at $T_{\mathrm{c}}$. To probe the nature of these diffraction anomalies we have carried out a neutron investigation of these PM effects that we report in the following sections.

\section{Experimental results.}

3.1 EXPERIMENTAL. - A single massive crystal of $\mathrm{KAlF}_{4}$ was grown with the [110] direction along the crystal growth axis by the Bridgman-Stockbarger 


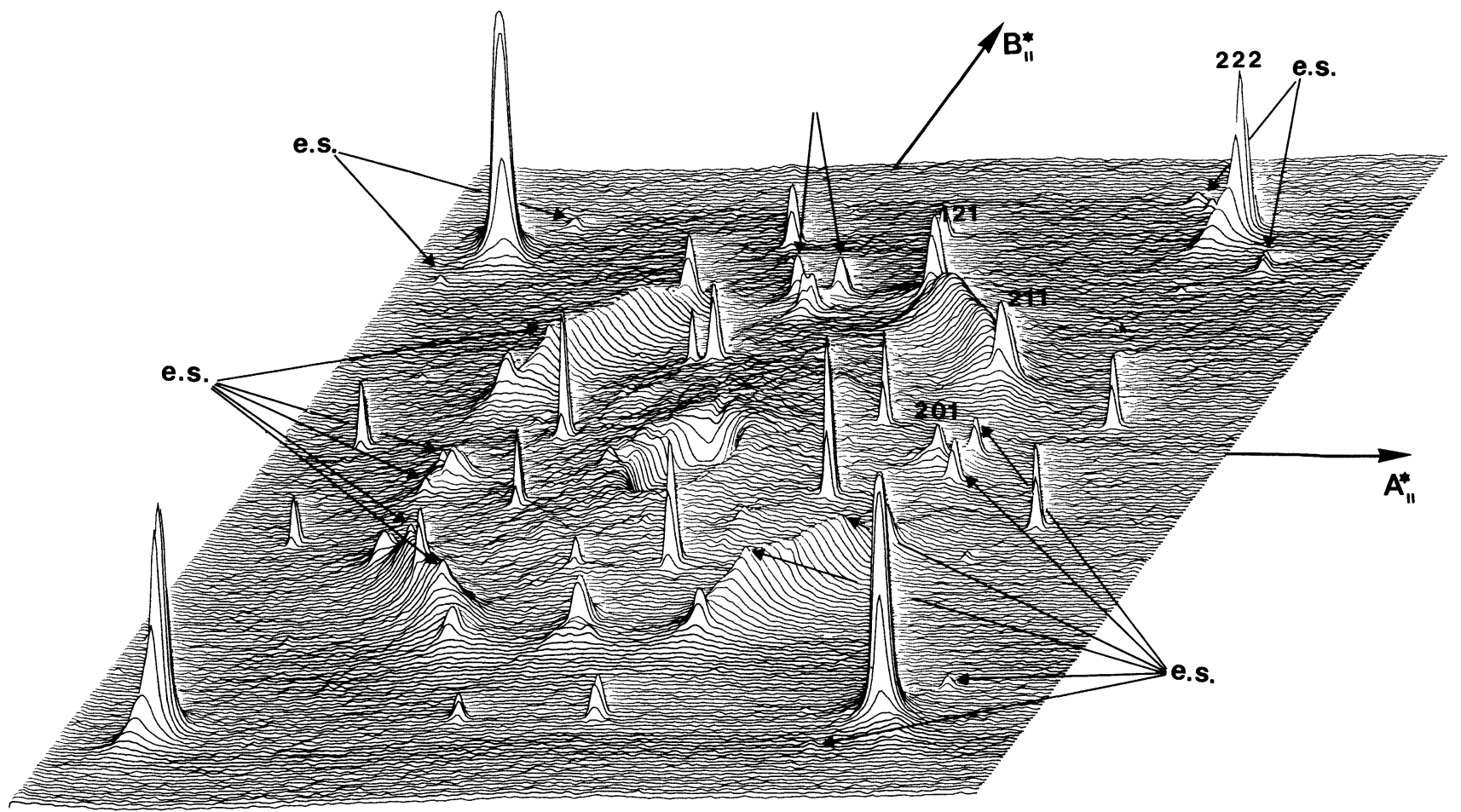

Fig. 3. - Three-dimensional representation of an X-ray mono-Laue photograph of the $\mathrm{KAlF}_{4}$ parent phase. Extra-spots are arrowed and DS located along the MX branch is obvious. The intensities are obtained by microdensitometry and the data are twice Fourier transformed and filtered to reduce the background (reflections which are not indexed or not arrowed arise from the $\lambda / 2$ contamination).

technique at P.E.C. laboratory (Université du Maine, Le Mans, France). From the massive bulk, a crystal $5 \times 5 \times 6 \mathrm{~mm}$ with (001) natural faces was cut with a diamond saw along the [110] and [1ํㅣ] directions.

Extra-spots were mapped out on the D10 four circle diffractometer of the I.L.L. (Grenoble, France). The crystal was mounted on a cadmium pin and inserted in an helium flow cryostat enabling a good sample temperature stability [17]. An absolute accuracy of $5 \times 10^{-2} \mathrm{~K}$ was obtained close to $T_{\mathrm{c}}$.

The $12^{\prime}$ divergent incident beam was reflected onto a focusing copper monochromator selecting the $1.26 \AA$ wavelength minimally affected by the $\lambda / 2$ contamination $\left(I(\lambda / 2) / I(\lambda)<4 \times 10^{-4}\right)$. A pyrolitic graphite analyser (004) was used to obtain a $0.1 \mathrm{THz}$ energy resolution.

The resolution function was experimentally determined by scanning the closest Bragg reflections of the measured extra-spots. A typical average resolution FWHM was $6 \times 10^{-3} \AA^{-1}$ along $Q_{\|}$direction of the horizontal plane and $10^{-2} \AA^{-1}$ along the perpendicular direction in the scattering plane.

Following the evidence of antiphase domains in $\mathrm{KAlF}_{4}$ by $\mathrm{X}$-ray and neutron powder diffraction studies [4], the anisotropy of the $(h+k=2 n+1$, $l \neq 0$ ) Bragg reflections (characteristic of the equatorial fluorine network) was investigated. In spite of the good $Q$-resolution no enlargment was observed. As a result, antiphase domains responsible for this effect were not present (or in minute proportions, i.e. with an effective size greater than $750 \AA$ ) in this crystal. This is a signature of the crystal quality and it can be suggested that antiphase domains arise in $\mathrm{KAlF}_{4}$ from the pulverizing procedure.

3.2 ElASTIC NEUTRON RESUlTS. - To determine more accurately the main characteristics of the observed extra-spots, the reciprocal space has been mapped out in the directions where extra-spots were expected to be found from the X-ray mono-Lauë patterns. It was found that :

- extra-spots are not in commensurate positions (as shown in Tab. II) with respect to the tetragonal lattice and these positions correspond to $q$ wavevectors which differ somewhat from one Brillouin zone to one another ;

- the extra-spots width is anisotropic as evidenced in figure $4:$ in the (110) plane a broadening is always observed in the $[1 \overline{1} 0]_{\mathrm{II}}$ direction whereas extra-spots are resolution limited along the $[110]_{\text {II }}(*)$. In the $[001]$ direction, reflections are not

$\left(^{*}\right)$ The direction of the broadening is inverted in (110) plane with regard to the apparent tetragonal symmetry. 
Table II. - Review of the PM extra-spots which were used in the refinement procedure to determine the lattice parameters of the PM phase. All these spots are belonging to a selected single domain. The extra-spots indices obtained in the tetragonal cell are converted into monoclinic indices prior to the refinements.

Tetragonal indices

$\begin{array}{rrr}-0.150 & 0.150 & 0.990 \\ -0.462 & 0.462 & 2.989 \\ -0.610 & 0.610 & 3.995 \\ -0.705 & 0.705 & -1.994 \\ 1.541 & 2.461 & 2.994 \\ 0.128 & 3.872 & -0.994 \\ -1.882 & 1.882 & -0.994 \\ 0.846 & 1.154 & 0.998 \\ 1.846 & 2.154 & 0.998 \\ 0.300 & 1.700 & -1.994\end{array}$

Pseudoorthorhombic indices

$\begin{array}{rrr}0 & 0 & 2 \\ 0 & 0 & 6 \\ 0 & 0 & 8 \\ 1 & 0 & -3 \\ 0 & 4 & 6 \\ 2 & 4 & 0 \\ 2 & 0 & 0 \\ 0 & 2 & 2 \\ 0 & 4 & 2 \\ 1 & 2 & -3\end{array}$

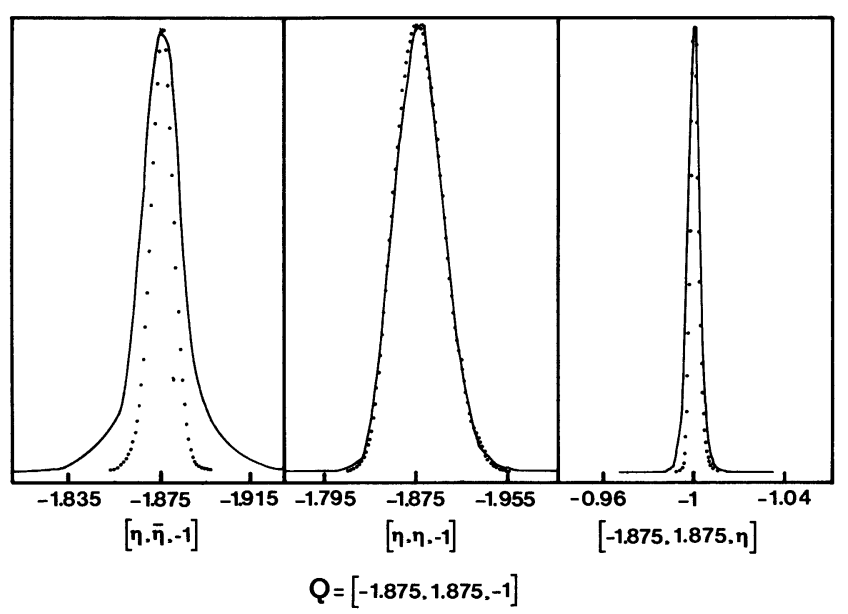

Fig. 4. - Experimental line profile shapes of extra-spots for variant $\mathrm{n}^{\circ} 1$ at position $Q(-1.875,1.875,-1)$ showing the enlargements along the [110] and [001] directions. The resolution function (dotted lines) was measured at position $(-2,2,-1)$.

resolution limited but present a smaller enlargement than in the $[1 \overline{1} 0]_{\text {II }}$ direction;

- the intensity and the $\mathbf{q}$ wavevector of the most intense extra-spots, namely the $-1.875,1.875$, -1 whose integrated intensity is approximately 4/10 000 of the closest Bragg peak integrated intensity, is temperature independent in the range $T_{\mathrm{c}}+5 \mathrm{~K}-T_{\mathrm{c}}+30 \mathrm{~K}$;

- the $\mathbf{q}$ wavevector of extra-spots located around the 001 parent phase Bragg reflections is as shown in figure 5 a multiple of the $l$ Miller index such as :

$$
\mathbf{q}=0.152(2) l\left( \pm \mathbf{a}^{*} \pm \mathbf{b}^{*}\right)
$$

— in addition this effect is also observed around

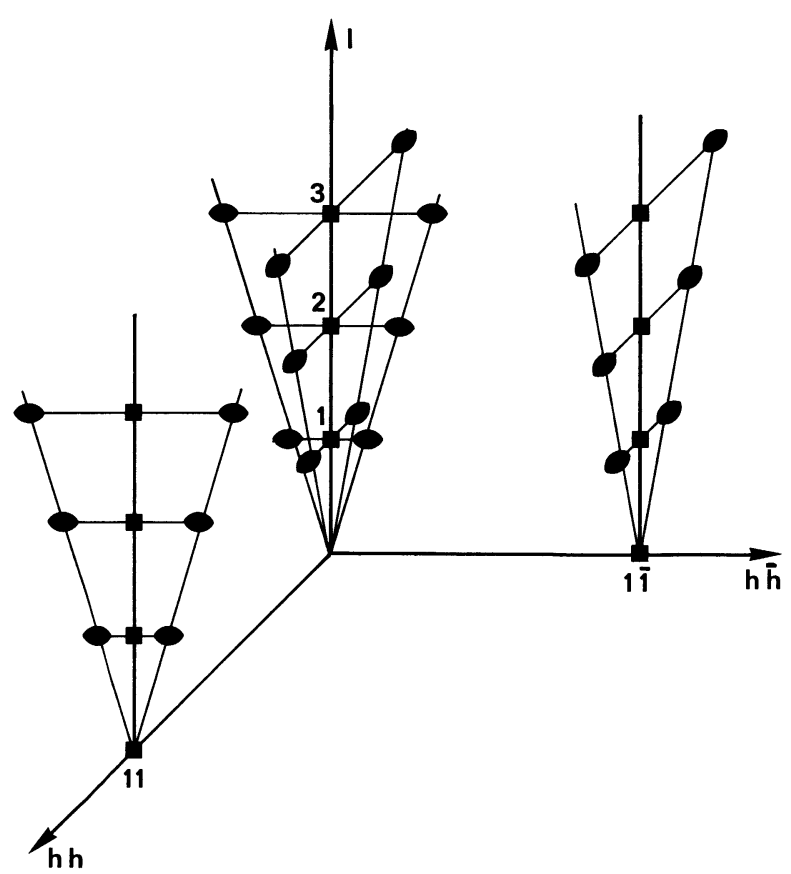

Fig. 5. - Three-dimensional representation of the extraspots (in elliptic shape) surrounding the $h h l$ and $h \bar{h} l$ Bragg reflections (in full squares) of the parent phase showing the $l$ dependence of the $\mathbf{q}$ wavevector and the direction of the broadening.

the $h h l$ or $h \bar{h} l$ parent phase Bragg reflections but with a $\mathbf{q}$ wavevector dependence :

$$
\begin{array}{ll}
\mathbf{q}= \pm 0.152(2) l\left(\mathbf{a}^{*}-\mathbf{b}^{*}\right) & \text { for } h h l \\
\mathbf{q} \equiv \pm 0.152(2) l\left(\mathbf{a}^{*}+\mathbf{b}^{*}\right) & \text { for } h \bar{h} l
\end{array}
$$

To a large extent, these results are quite reminiscent of those observed in the so called PM phase of metallic alloys in which they are the object of controversy. Under these circumstances, these effects may be attributed either to an incommensurate phase or to diffraction by an additional phase called the PM phase coexisting with the parent phase.

In $\mathrm{KAlF}_{4}$, the similarity existing between the Xray mono-Laue pattern of the product and of the PM phases together with the above temperature independent scattering features seem to rule out the incommensurate phase picture and strongly suggest that extra-spots arise from domains (or variants) inserted in the parent host matrix. They do not appear to be related to thermal fluctuations since the scattering does not depend upon temperature. These variants are expected to have a somewhat similar structure and arrangement (described in Sect. 2) to that of the product phase since they produce nearly the same diffraction effects.

The key consideration which evidences that the variant picture can be considered as confidently appropriate is the $l$ dependence of the $\mathbf{q}$ wavevector of extra-spots surrounding the $h h l$ parent phase Bragg reflections. Indeed this observation consistent- 
ly proves that these extra-spots lie in planes which make a $\delta$ angle with the $c_{\mathrm{II}}^{*}$ axis of the parent phase such as :

$$
\operatorname{tg} \delta=0.152 a_{\mathrm{II}}^{*} \sqrt{2} / c_{\mathrm{II}}^{*}
$$

giving $\delta=14.7^{\circ}(2)$.

Around the 001 parent phase Bragg reflections, the apparent fourfold symmetry which arises from the presence of extra-spots lying along four axes can be significantly attributed to four variants set out at $90^{\circ}$ from each other.

For each variant let these axes be the $c_{\mathrm{PM}}^{*}$ reciprocal axis of the PM phase. As extra-spots are within the (001) planes of the parent phase it turns out that a simple lattice correspondence can be established between the PM and the parent phases reciprocal lattice parameters (RLP) $c_{\mathrm{PM}}^{*}$ and $c_{\mathrm{II}}^{*}$. As in addition some of the extra-spots (in Fig. 6) are located within the $l / 2$ reciprocal planes of the parent phase it can be deduced that the correspondence is :

$$
c_{\mathrm{PM}}^{*}=c_{\mathrm{II}}^{*} / 2 \cos \delta \text {. }
$$

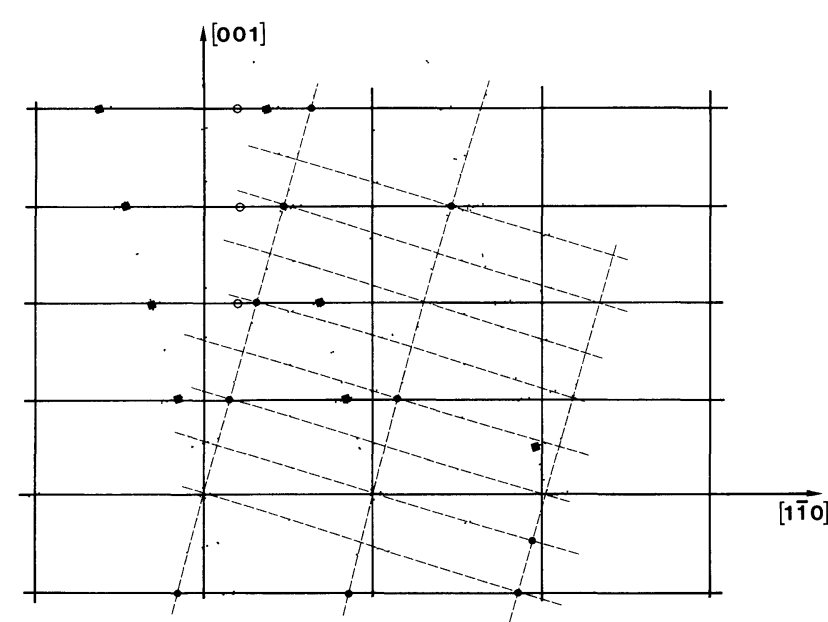

Fig. 6. - Cross-section of the (110) scattering plane showing the superposition of the tetragonal reciprocal lattice (full lines) together with two variants of the monoclinic lattice ; extra spots belonging to variant $n^{\circ} 1$ (in dashed lines) are in full circles and extra-spots belonging to variant $\mathrm{n}^{\circ} 2$ (in doted lines) are in full squares. Extra-spots in open circles are not explained in the variant framework (see text).

As illustrated in figure 6 , the investigation of extra-spots belonging to the (110) plane has confirmed the variants description. In this plane, extraspots are expected to arise from only two variants (except for large $l$ Miller indices where some of them may come from the two others). It is observed that extra-spots are definitely arranged at the corners of two superimposed periodic reciprocal lattices defined by the above $c_{\mathrm{PM}}^{*}$ RLP and an $a_{\mathrm{PM}}^{*}$ RLP which is $17^{\circ}$ off from the $[1 \overline{1} 0]$ direction of the parent phase and $92^{\circ}$ off from the $c_{\mathrm{PM}}^{*}$ axis. The $a_{\mathrm{PM}}^{*}$ RLP is found to be roughly equal to :

$$
a_{\mathrm{PM}}^{*}+=a_{\mathrm{II}}^{*} \sqrt{2}=a_{\mathrm{I}}^{*} .
$$

In addition, the $17^{\circ}$ angle evidences that the PM phase lattice is rotated around the [110] direction of the parent phase exactly as it is the case in the « martensitic » phase of $\mathrm{KAlF}_{4}$.

The last RLP has been obtained by measuring the position of extra-spots which were outside the (110) plane. It has been found that the $b_{\mathrm{PM}}^{*}$ RLP is perpendicular to the (110) plane and corresponds to :

$$
b_{\mathrm{PM}}^{*}=a_{\mathrm{II}}^{*} / \sqrt{2}=a_{\mathrm{I}}^{*} / 2
$$

To complete these informations, it must be added that in the (110) plane three spots (in open circles in Fig. 6) remain unexplained in the above framework. These spots are located around the 001 reflections of the parent phase with the constant $q$ wavevector $\mathbf{q}=0.25\left(\mathbf{a}^{*}-\mathbf{b}^{*}\right)$. It was checked that their intensity and wavevector were also temperature independent and we presume that these spots might arise from multiple scattering since the reciprocal space is overcrowded.

As a result, these first measurements have shown that the PM phase of $\mathrm{KAlF}_{4}$ can be described by a monoclinic unit cell which has the following lattice parameters :

$$
\begin{array}{ll}
a_{\mathrm{PM}}=a_{\mathrm{I}} & b_{\mathrm{PM}}=2 b_{\mathrm{I}} \\
c_{\mathrm{PM}}=2 c_{\mathrm{I}} \cos \delta & \beta_{\mathrm{PM}}=87.67^{\circ} .
\end{array}
$$

To corroborate these results, we have carefully selected all the measured extra-spots belonging to a single variant (variant number 1 in Fig. 6). Taking into account the $17^{\circ}$ misorientation, we have established the correspondence existing between the indexation of these extra-spots in the tetragonal parent cell and their indexation in the monoclinic cell. From their angular positions the lattice parameters of the monoclinic PM phase were refined through the usual least-square procedure. The following lattice parameters were found:

$$
\begin{array}{ll}
a_{\mathrm{PM}}=3.643 \AA(3) & b_{\mathrm{PM}}=7.125 \AA(5) \\
c_{\mathrm{PM}}=11.922 \AA(8) & \beta_{\mathrm{PM}}=87.67 \AA(6) .
\end{array}
$$

Let us notice that according to the present investigation no reflection such as $h+l$ odd has been observed so that it is reasonable to assume a $B$ centred lattice. The real monoclinic cell would then have the following parameters :

$$
\begin{array}{ll}
a=3.643 \AA & b=7.125 \AA \\
c=12.313 \AA & \beta=104.67^{\circ} .
\end{array}
$$

3.3 Simulation OF THE X-RAY MONO-LAUË PATTERN. - With the lattice parameters of the PM 
phase known, it was tempting to simulate the X-ray mono-Lauë pattern of the room temperature phase of $\mathrm{KAlF}_{4}$ according to the above predicted arrangement (i. e. superposition with the tetragonal parent phase of four monoclinic variants dispatched at $90^{\circ}$ from each others and rotated from $17^{\circ}$ around the $b_{\mathrm{PM}}^{*}$ axis). The results presented in figure 7 are totally conclusive.

At the light of these results, it is now valuable to search for an interpretation of the PM and parent

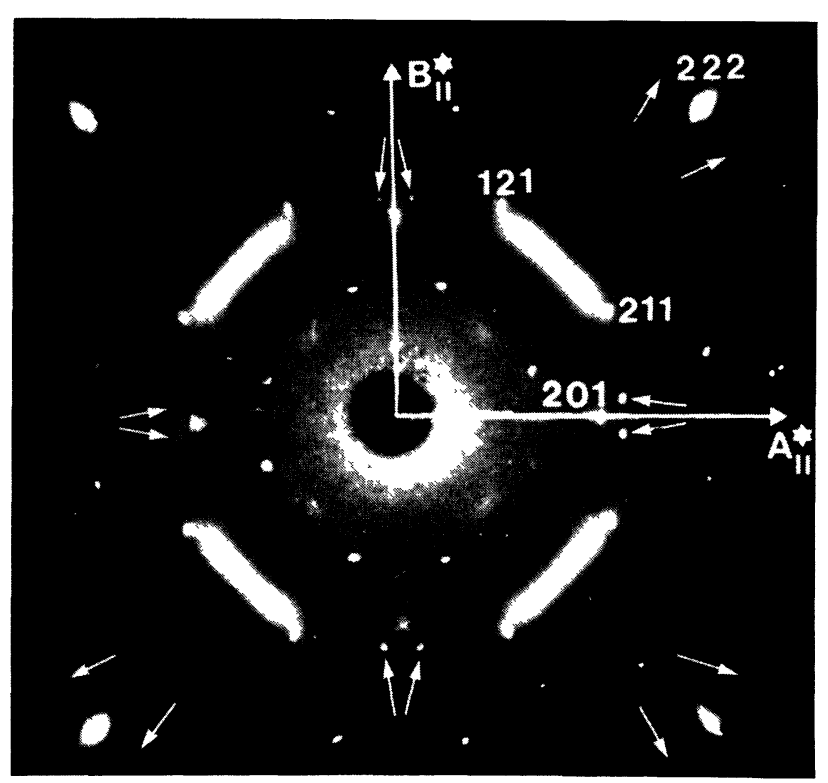

a)

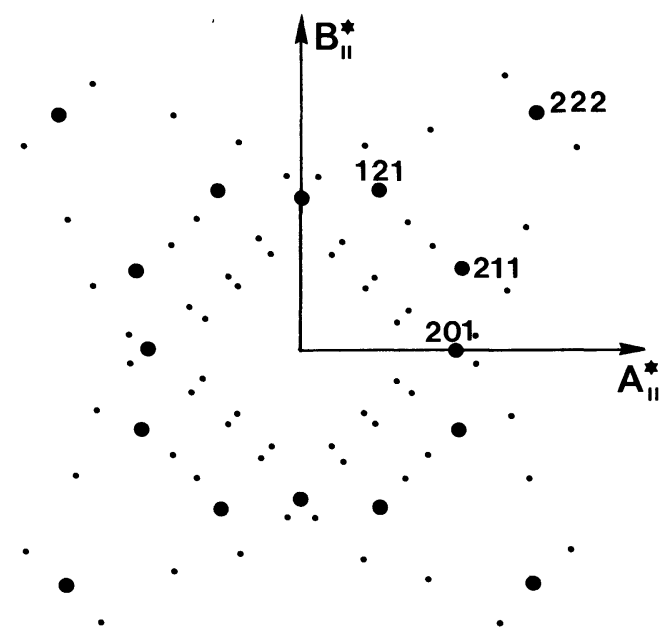

b)

Fig. 7. - a) X-ray mono-Laue photograph of the $\mathrm{KAlF}_{4}$ parent phase with the tetragonal $c$ axis perpendicular to the photograph and a fixed crystal with the $\mathrm{Cu}$ $\mathrm{K} \alpha$ radiation (visible extra-spots are arrowed) ; b) simulation of this photograph according to the predicted arrangement. $\mathrm{A} \pm 2^{\circ}$ scatter about the horizontal and vertical axes are used to account for the low resolution of the X-ray instrument together with the large mosaicity of cleaved crystals. phases coexistence. For simplicity, we shall refer in the following to a single variant (variant $n^{\circ} 1$ in Fig. 6).

\section{On the PM phase structure of $\mathrm{KAlF}_{4-}$.}

4.1 The PM PHASE Structure. - The above results have consistently demonstrated that extraspots observed in $\mathrm{KAlF}_{4}$ at room temperature can be related to variants of a PM phase inserted in the tetragonal parent host matrix. As a consequence this rules out the incommensurate phase picture but at the same time raises the problem of determining how these two phases can coexist and accomodate without disturbing the apparent optical homogeneity of the crystals. It emerges that to correctly sort out this question it is now necessary to foresee what may be the structure of the PM phase. Indeed the experimental determination of this structure is particularly difficult since the integrated intensity of extra-spots is several orders of magnitude less than that of the tetragonal parent phase. Furthermore, many of them are superimposed on the parent phase reflections and the number of reflections having a reliable intensity is too small to permit a structural determination. Moreover, the further we are from the origin of reciprocal space the more the diffraction lines can overlap.

Be that as it may, we propose a possible structure based upon :

- the knowledge of the lattice parameters of the PM phase together with the orientation relations presented by the two phases ;

- the rigidity of $\mathrm{AlF}_{6}^{3-}$ octahedra and the assumption that the octahedra sheets form continuous layers for which the interlayer cohesion is much less than the intralayer one as evidenced from the macroscopic properties examination of all the $\mathrm{ABX}_{4}$ compounds and from the recent study of the lattice dynamics of the tetrafluoroaluminates $[2,6]$;

- the similarity existing between the X-ray mono-Lauë of the PM and product phases which suggests that the PM atomic arrangement must be very similar to that of the product phase.

Let us first consider the orientation relation which exists in direct space between the monoclinic cell and the ideal tetragonal one. Figure 8 illustrates the passage from the reciprocal cell to the direct one and shows that the monoclinic " $a_{\mathrm{PM}}$ " axis is turned by $14.7^{\circ}$ around the $[010]_{\mathrm{I}}$ direction. As a direct consequence, this means that the octahedra sheets present an inclination of $14.7^{\circ}$ at the parent-PM phases interface. This is illustrated in figure 9 in which we have inserted the monoclinic unit cell into the parent host matrix with the $14.7^{\circ}$ inclination. Assuming that the continuity of the octahedra sheets stands for all sheets which is the sine qua non condition for the 


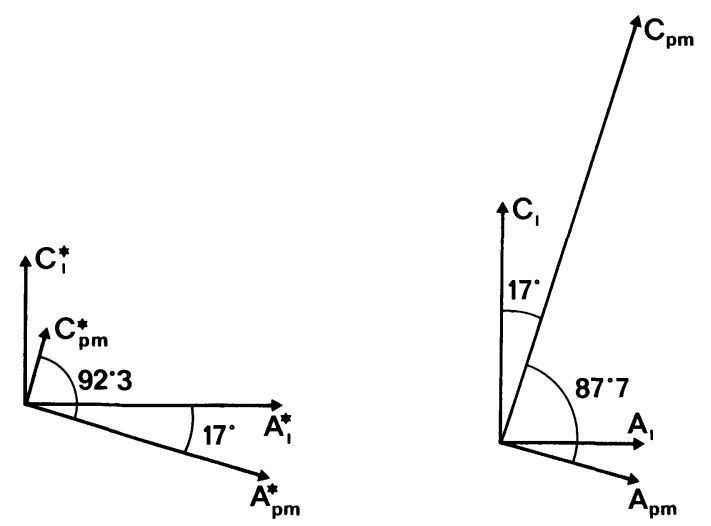

Fig. 8. - Correspondence between the direct and reciprocal axes for variant $n^{\circ} 1$.

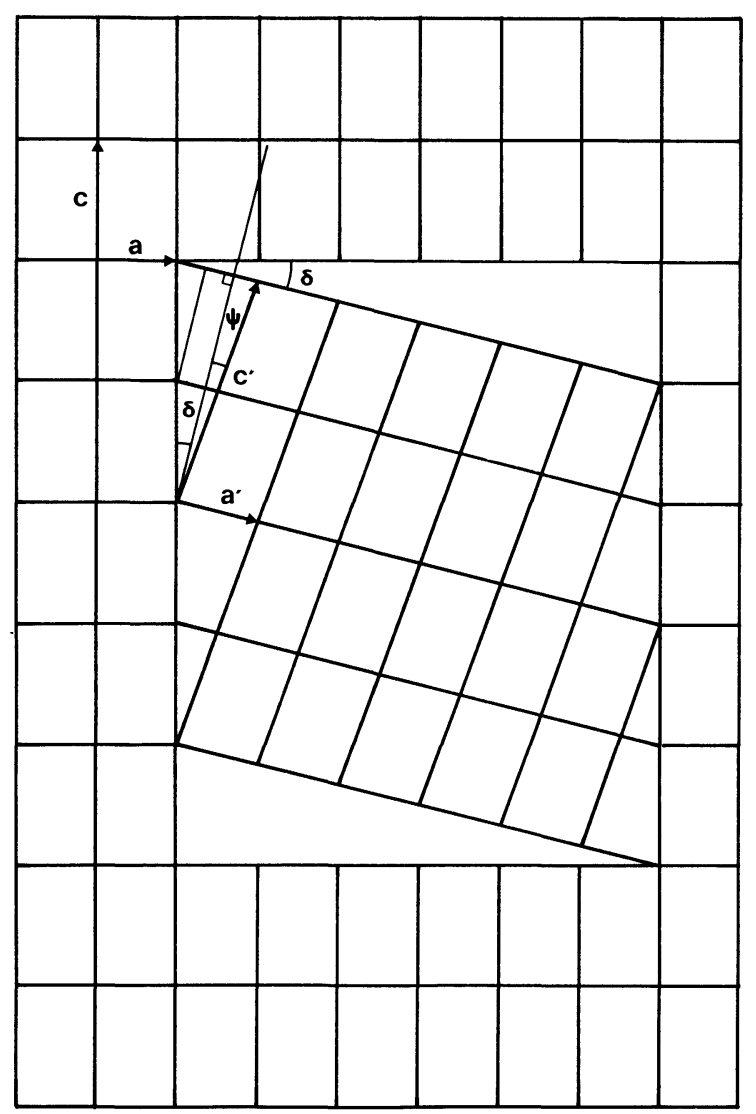

Fig. 9. - Representation of the phases accommodation for the variant $n^{\circ} 1$ with the edge dislocations which are involved.

perfect accomodation between the two phases, it can be seen that adjacent octahedra sheets are displaced in the PM phase by:

$$
c_{\text {II }} \sin \delta=1.56 \AA
$$

with respect to their positions in the parent phase. As in the product phase, this value is very close to half the $a_{\mathrm{I}}$ parameter. Consequently since the oc- tahedra network is very similar in the product and PM phases it clan be inferred that the $\mathrm{K}^{+}$ions settle between the sheets as in the product phase. To predict the PM phase structure, let us now consider the tilting situation in the PM phase. According to reference [3] it can be deduced that twice the sum of the ionic radii of the $\mathrm{Al}$ and $\mathrm{F}_{\mathrm{eq}}$ ions is respectively :

$$
\begin{aligned}
& d_{1}=3.64 \AA \text { in the parent phase at } 300 \mathrm{~K} \\
& d_{2}=3.68 \AA \text { in the product phase at } 4 \mathrm{~K} .
\end{aligned}
$$

Moreover in each phase such distances are shown to be quite temperature independent [2]. With this information and the $a_{\mathrm{PM}}$ and $b_{\mathrm{PM}}$ values, the octahedron tilts around the different PM phase axes can be deduced as follows :

$$
\begin{aligned}
& \Phi_{001 \mathrm{PM}}=\arccos a_{\mathrm{PM}} / d_{2}=8^{\circ} \\
& \Phi_{100 \mathrm{PM}}=\arccos b_{\mathrm{PM}} / 2 a_{\mathrm{PM}}=12^{\circ} .
\end{aligned}
$$

These values indicate that the PM phase structure is the same as the product one which belongs to the $\mathbf{P} 2 / \mathrm{m}$ space group. In addition this shows that owing to a non zero rotation angle around the [001] direction, the $a_{\mathrm{PM}}$ lattice parameter must be doubled; the cell parameters are then more probably :

$$
\begin{array}{ll}
a_{\mathrm{PM}}=7.286 \AA & b_{\mathrm{PM}}=7.125 \AA \\
c_{\mathrm{PM}}=11.922 \AA & \beta_{\mathrm{PM}}=87.67^{\circ}
\end{array}
$$

in the pseudo-orthorhombic cell,

$$
\begin{array}{ll}
a_{\mathrm{PM}}=7.286 \AA & b_{\mathrm{PM}}=7.125 \AA \\
c_{\mathrm{PM}}=12.313 \AA & \beta_{\mathrm{PM}}=104.67^{\circ}
\end{array}
$$

in the monoclinic cell.

The above results clearly indicate that the product and PM phases structures are identical. Nevertheless, the PM phase lattice parameters significantly differ from the product phase ones by a larger monoclinic distortion $\left(2.3^{\circ}\right.$ instead of $\left.0.18^{\circ}\right)$ and different amplitudes in the tilt angles. This is the signature of the stress induced by the parent phase onto the PM one. This point will be discussed in section 4-3.

\subsection{Relative Volumes OF THE PM AND PARENT} PHASES. - Considering that the PM and the product phases have the same structural arrangement, it can be assumed that the diffraction lines intensities of both phases are probably not significantly different. According to the neutron powder investigations of the parent and product phases [3], the ratio of intensities of a reflection in the parent and product phases can be determined. It is then possible to estimate the respective volumes of the PM and product phases. In this crystal, this ratio is on average $2.0(0.5) \times 10^{-4}$ for a single PM domain and thus $8(2) \times 10^{-4}$ for the four domains. As a 
result the volume of the PM phase in the $5 \times 5 \times$ $6 \mathrm{~mm}^{3}$ crystal is roughly $0.12 \mathrm{~mm}^{3}$. This explains why the PM phase reflections are so difficult to observe.

4.3 The ACCOMOdation OF THE TWO PHASES. As illustrated in figure 10 , the accomodation is achieved by means of a dislocation-mediated process which is consistent with a martensitic transformation. The two phases fit together along undistorted (110) contact planes which can be considered as the habit plane of the coexisting phases. The above orientation relation together with the lattice parameters entirely determine the contact between the

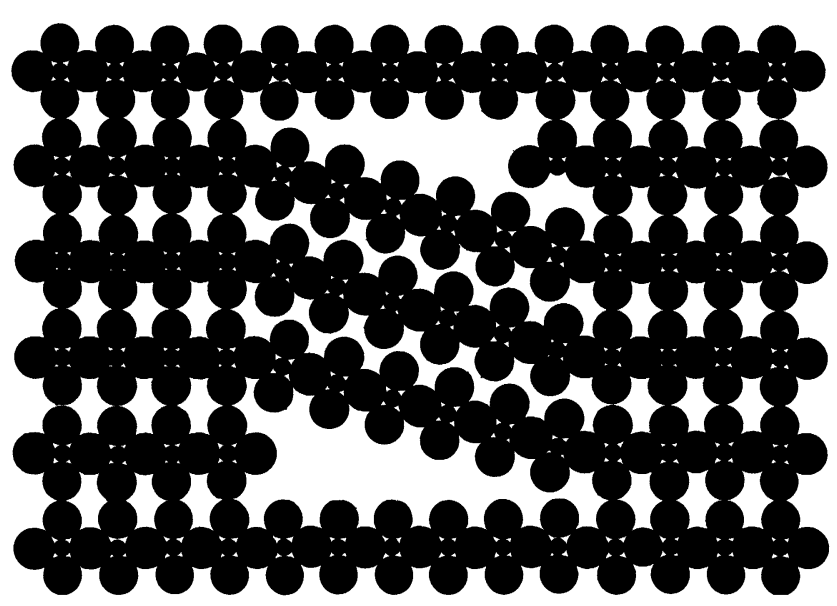

Fig. 10. - Schematic representation of the $\mathrm{AlF}_{6}$ octahedra sheets in the parent and PM phases with evidence of the accommodation. The relative volumes of the ions are respected and only the ions which do not overlap have been drawn. The distortion of the parent phase lattice in the vicinity of the PM phase together with the ionic arrangement in the «vacancies» are not taken into account.

two phases. In particular, the accommodation is governed by the geometrical relation :

$$
p a_{\mathrm{PM}} \sin \delta=n c_{\mathrm{II}} \quad \text { with } n \text { and } p \text { integers }
$$

which is correctly satisfied for a $n / p$ ratio approximately equal to 0.15 . Such a ratio is difficult to obtain precisely for several reasons :

- first, it is very likely that the dislocations produce strains in the parent phase lattice in the neighbourhood of the PM phase so that the $c_{\text {II }}$ lattice parameter which is necessary to perform the calculation is not known;

- secondly, the extra-spot widths are not resolution limited which probably means that several couples $(n, p)$ can be suitable to satisfy the above equation.
Nevertheless, if we consider an average 0.15 ratio consistent with the couple $(p, n)$ equal to $(20,3)$, this shows that the PM phase of $\mathrm{KAlF}_{4}$ is formed of embryos which are size limited in the [110] direction by contact planes. The quantity $p=20$ obtained from geometrical considerations suggests that embryos are approximately $70 \AA$ wide along the [110] direction. No size limitation is expected in the [110] direction whereas along the [001] direction the embryos size is determined by the position of edge dislocations. As shown in figure 4, these results are fully in agreement with the anisotropy of the extraspots line-widths along these directions. The correlation lengths $\xi$ along the above directions have been determined by using the Warren-Averbach formalism based upon the Fourier analysis of the line profile shapes [18]. The idea to use such a formalism is guided by the fact that the suitable functional form of the scattering is less certain. As a result, the measured line profile shape $h(\mathbf{q}, \mathbf{Q})$ and the measured resolution function $g(\mathbf{q}, \mathbf{Q})$ have been described by their Fourier coefficients $\left(A_{n}^{h}, B_{n}^{h}\right)$ and $\left(A_{n}^{g}\right.$ and $\left.B_{n}^{g}\right)$. The measured line profile is the convolution of the resolution function with the true sample size-dependent line shape. With nearly symmetrical profile shapes, the true sample size is given by :

$$
\lim _{n \rightarrow 0} \mathrm{~d} A_{n}^{s} / \mathrm{d} n=\lim _{n \rightarrow 0} \mathrm{~d} A_{n}^{h} / \mathrm{d} n-\lim _{n \rightarrow 0} \mathrm{~d} A_{n}^{g} / \mathrm{d} n .
$$

This leads to the following correlation lengths :

$$
\begin{aligned}
& \xi_{110}=100 \pm 30 \AA \\
& \xi_{001}=1000 \pm 200 \AA .
\end{aligned}
$$

Such values are in fairly good agreement with the $n$ value. Nevertheless the $A_{n}$ are not linear with $n$; this shows that there is a size distribution for the embryos.

At this point it is now worthwhile to note that the main features of the PM phase can be deduced from the accomodation scheme alone and more especially from the $(n, p)$ couple. If we denote by a prime the lattice parameters of a PM phase for a given $(n, p)$ couple of integers and if we use $a=a_{\mathrm{II}} / \sqrt{2}$ and $c$ the lattice parameters of the parent phase, the following parameters can be calculated :

$-\delta^{\prime}$ angle between $\mathbf{a}$ and $\mathbf{a}_{\mathrm{PM}}^{\prime}$

$$
\delta^{\prime}=\arctan (n c / p a)
$$

- $\psi^{\prime}$ angle representing the deviation from a perfect alternated octahedra sheets arrangement as occurs in the ideal structure from which the PM and product phases are derived :

$$
\psi^{\prime}=\arctan (a / 2 c+(n / p-2) n c / 2 p a)
$$


- $a_{\mathrm{PM}}^{\prime}$ parameter

$$
a_{\mathrm{PM}}^{\prime}=\left(a^{2}+(n c / p)^{2}\right)^{1 / 2}
$$

- $d^{\prime}$ interlayer distance

$$
d_{\mathrm{PM}}^{\prime}=c /\left(1+(n c / p a)^{2}\right)^{1 / 2} .
$$

Since both the parent and product phases are stable at room temperature, a phase with the structure close to the latter can be included in the former provided that for a couple $(n, p)$ of integers $\psi^{\prime}$ will be as close as possible to zero and $a_{\mathrm{PM}}^{\prime}$ less than twice the AlF distance observed in the $\mathrm{KFeF}_{4}$ ideal structure, i.e. $3.68 \AA$. Figure 11 enables to show that these conditions are almost satisfied for the couple $(n, p)$ equal to $(2,13)$ or $(3,20)$ and every greater integers $(n, p)$ such as $n / p$ belonging to $[3 / 20,2 / 13]$. Smaller $n / p$ ratios would give rise to a greater monoclinic distortion and greater ratios to too large $a_{\mathrm{PM}}^{\prime}$ parameters. Thus it appears that the reality results from the competition between two competing conditions and that the large monoclinic distortion can be regarded as the consequence of the accomodation scheme. It can be noted that when $p / n$ belongs to $[3 / 20,2 / 13], \delta^{\prime}$ belongs to $\left[14.87^{\circ}\right.$, $\left.14.52^{\circ}\right]$ and $d^{\prime}$ to $[5.94 \AA, 5.95 \AA]$ which are values close to the experimental ones. On the contrary the $a_{\mathrm{PM}}$ parameter is found smaller than $a_{\mathrm{PM}}^{\prime}$. This discrepancy which is the consequence of the octahedra tilting around the [001] axis in the PM phase is not so surprising since it can be reasonably inferred that the parent phase matrix is probably distorted in the vicinity of the embryos. The two parameters would be identical if we consider in the parent phase a local $a=3.52 \AA$ parameter which corresponds to an octahedra tilt angle of $15^{\circ}$ instead of $11^{\circ}$ (such a parameter does not significantly modify the above results).

The presence in the parent phase of such embryos which are non thermally activated seems to confirm the idea of preexisting subcritical embryos with a semi-coherent interface developed by Cohen [19] and Christian [20] (let us note that such embryos have been already observed in metals [21]). Furthermore, the $\mathrm{KAlF}_{4}$ embryo description also involves the presence of edge dislocations on both sides of the PM phase as proposed by Easterling and Thölen [22]. It can be inferred that these dislocations mainly arise during the layer by layer crystal growth and are responsible for the presence of the PM embryos.

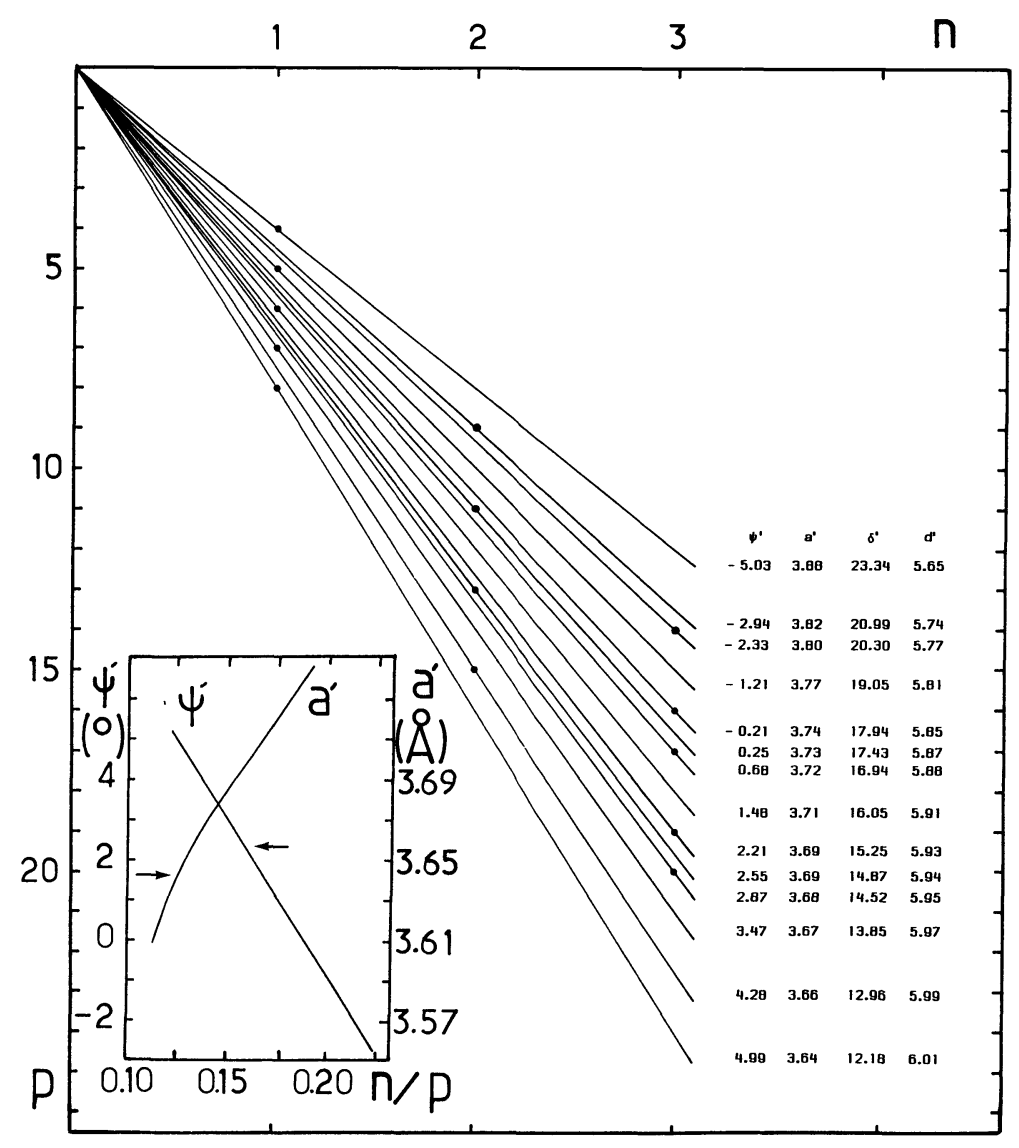

Fig. 11. - Illustration of the parameters $a^{\prime}, \psi^{\prime}, \delta^{\prime}$ and $d^{\prime}$ for different $(n, p)$ couples. The inset shows the $\psi^{\prime}$ and $a^{\prime}$ dependence as a function of the $n / p$ ratio and evidences the experimental situation $\psi^{\prime}=2.3^{\circ}$ and $a^{\prime}=3.643 \AA$ (arrowed in the pattern). 
The dislocations can be either stress-induced and purely intrinsic or mediated by point defects and to have therefore an extrinsic origin. The latter point has been evidenced since the introduction of controlled point defects tremendously favour the $\mathrm{KAlF}_{4} \mathrm{PM}$ phase coexistence with the tetragonal parent phase. The study of this effect is presently in progress. Let us also note that the introduction of $\mathrm{Rb}$ in $\mathrm{KAlF}_{4}$ impedes the direct martensitic transformation and leads to an intermediate phase which is observed on a temperature range depending on the $\mathrm{Rb}$ concentration and which eventually transforms to the product phase [23]. To comment on the former point it can be added that the cleavage procedure may also favour the creation of additional nucleating dislocations. The application of stress in $\mathrm{KAlF}_{4}$ crystals nearly free of dislocations (as it was presumably the case for crystal I in paper 5) might provide an answer to this question.

\section{Discussion.}

An additional question arises from the role played by the embryos in the onset of the martensitic transformation. As their sizes and number are temperature independent at least above $T_{\mathrm{c}}+5 \mathrm{~K}$, it can be reasonably inferred that embryos are stuck in between non-glissile interfaces which impede their development on cooling. As a result, the question is to understand how the embryos should be suddlenly responsible for the starting of the martensitic transformation whilst they do not evolve over a wide range of temperature above $T_{\mathrm{c}}$ ?

According to Cohen [19] who predicted the presence of preexisting embryos in martensitic transformation, the transition occurs when the difference of energy between the parent and product phases becomes so negative that the embryos become hypercritical and thus constitute active nuclei for the transformation. However, this idea which can be regarded as confidently appropriate from a thermodynamical point of view is difficult to evidence from an experimental one because the transformation is extremely quick and destructive. Such an idea has been developed by $\mathbf{R}$. Bruisma and $\mathrm{A}$. Zangwill to interpret the subsequent transformation of pseudomorphic phases in epitaxial overlayers [24]. Such phases are very similar to the embryos observed in the PM phase of $\mathrm{KAlF}_{4}$. These authors underline that the pseudomorphic phase always competes with the bulk stable phase for ultimate stability to satisfy the demands of the parent phase thermodynamics. In their constructed phase diagram, they emphasize the martensitic nature of the transformation of the pseudomorphic phase for sufficiently thick overlayers.
On the other hand, it is also proposed that the critical stage would be the propagation of interface dislocations which are necessary to observe the embryos [25]. As shown above, it is clear from a structural point of view and from the experimental results that the interface in $\mathrm{KAlF}_{4}$ is non-glissile above $T_{\mathrm{c}}+5 \mathrm{~K}$ so that this explanation does not appear to be fully convenient.

Another alternative to interpret the onset of the transformation might be provided by the existence of the flat MX branch of low frequencies phonons which necessarily create instabilities in the tetragonal parent host matrix $[2,5,6]$. Let us first recall that our experimental results indicate that these phonons are not responsible for the extra-spots existence in the PM phase. Nevertheless, such phonon instabilities are frequently observed in metallic alloys which undergo the martensitic transformation and which exhibit a PM phase [26] (in metallic alloys the phonon anomalies are yet observed along acoustic branches). Similarly to $\mathrm{KAlF}_{4}$, the phonon dispersion curves in metallic alloys do not present any real minimum but only show a saddle-point. This point indicates that phonons in martensitic transformation do not behave like in displacive transitions in which the slowing down of a particular soft optic mode is responsible for the transition. This point was recently evidenced in the comparative study of the two isomorphous compounds $\mathrm{RbAlF}_{4}$ and $\mathrm{KAlF}_{4}$ which undergo both transitions $[2,5]$. In $\mathrm{RbAlF}_{4}$, the diffuse scattering (DS) also located along the MX branch and originating from soft modes becomes more and more intense when $T_{c}$ is closely approached and eventually condenses at the $\mathrm{X}$ point at $T_{\mathrm{c}}$ as expected from the soft mode theory [27]. On the contrary, in $\mathrm{KAlF}_{4}$ the DS anisotropy remains the same close to $T_{\mathrm{c}}$. This strongly suggests that the $\mathrm{KAlF}_{4}$ martensitic transformation is not preceeded by the ultimate condensation of a particular soft mode probably because the tetragonal lattice becomes too unstable. The reason of this instability could be the presence of the stressed embryos which might act as a driving force at $T_{\mathrm{c}}$ when they become hypercritical. Nevertheless one cannot think that the soft phonons do not play a major role in the onset of the martensitic transformation. Thus, the next stage in the understanding of the $\mathrm{KAlF}_{4}$ martensitic transformation now implies the knowledge of the eigen modes which are responsible for the DS observed along the MX branch. This work will be reported soon $[2,6]$.

Finally another possibility to interpret the transformation could be the coupling of the vibrational modes with moving dislocations in small limited regions or shear bands as proposed by C. S. Coffey [28] since when the velocity of the dislocations is high enough, it seems that destructive transitions can occur. 


\section{Conclusion.}

In this study, we have demonstrated that the diffraction anomalies observed in the parent phase of $\mathrm{KAlF}_{4}$ can be attributed to non-thermally activated variants of a PM phase which coherently coexists with the tetragonal parent phase. The PM phase structure is put forward on the bases of geometrical considerations and of the continuity of octahedra layers at the phases interface. A possible explanation based upon a dislocation mediated process is proposed to account for the accomodation of the two phases. Thus it is shown that the PM phase is composed of embryos which are very similar to the pseudomorphic phase observed in epitaxial overlayers. From the anisotropy of the extra-spots the embryos have been found to be size limited in the perpendicular directions to the dislocation line. In addition, the volume of the embryos has been shown to be extremely small (i.e less than $1 / 1000$ of the total crystal volume). Nevertheless, our results still pose the considerable theoretical challenge of explaining the mechanism of the transformation. In particular the exact role played by the embryos in the onset of the martensitic transformation is not yet clear especially when it seems that the major role can presumably be attributed to soft phonons.

\section{Acknowledgments.}

The authors are grateful to G. Niesseron for technical assistance in the crystal growth and to R. Chagnon for technical assistance on the D10 instrument. They wish to thank D. Taupin and F. Lecoeuvre from the Microdensitometry Department of C.N.R.S. and the I.L.L. for the neutron facilities.

\section{References}

[1] Nouet, J., Pannetier, J. and Fourquet, J. L., Acta Crystallogr. B 37 (1981) 32.

[2] Bulou, A., Thesis 1985.

[3] Launay, J. M., Bulou, A., Hewat, A. W., Gibaud, A., Laval, J. Y. and Nouet, J., $J$. Physique 46 (1985) 771.

[4] Gibaud, A., Le Bail, A. and Bulou, A., J. Phys. C 19 (1986) 4623.

[5] Gibaud, A., Bulou, A., Launay, C. and Nouet, J., J. Physique, in press.

[6] Bulou, A., Gibaud, A., Rousseau, M. and NOUET, J., to be published.

[7] KRIVEN, W. M., International Summer Course on Martensitic Transformations (1982) Heverlee Leuven Belgium.

[8] Wayman, C. M., Physical Metallurgy 3rd ed., Cahn, R. W. and Haasen, P. (Eds) (Elsevier Science Publishers) 1983, Chap. 15.

[9] Adda, Y., Dupouy, J. M., Philibert, J. and QUÉRÉ, Y., Eléments de Métallurgie Physique. Transformation par cisaillement, La Documentation Française (Ed), Tome IV, Chap. 31.

[10] Michal, G. M., Moine, P. and Sinclair, R., Acta Metall. 30 (1981) 125.

[11] Satija, S. K., Shapiro, S. M., Salamon, M. B. and Wayman, C. M., Phys. Rev. B 29 (1984) 6031.

[12] Shapiro, S. M., NodA, Y., FujII, Y. and Yamada, Y., Phys. Rev. B 30 (1984) 4314.

[13] Salamon, M. B., Meilche, M. E. and Wayman, C. M., Phys. Rev. B 31 (1985) 7306.
[14] Bansal, G. K. and Heuer, A. H., Acta Metall. 20 (1972) 1281 and 22 (1974) 409.

[15] Kennedy, S. W. and Fraser, W. L., Proc. Int. Conf. Solid-Solid Phase Transformations, Carnegie-Mellon Uni. (1981) 1539.

[16] Kaufman, L. and Cohen, M., Prog. Met. Phys. 7 (1958) 165.

[17] Zeyen, C. M. E. and Chagnon, R., Rev. Phys. Appl. 19 (1984) 789.

[18] Waren, B. E. and Averbach, B. L., J. Appl. Phys. 21 (1950) 595.

[19] Cohen, M., Trans. AIME 219 (1958) 175.

[20] Christian, J. W., Phase Transformations, in Physical metallurgy, 2nd Ed. R. W. Cahn (Ed) (North-Holland, Amsterdam) 1970, Chap. 10.

[21] Wilson, J. A. and De Podesta, M., J. Phys. F. 16 (1986) L121.

[22] Easterling, K. E. and Thölen, A. R., Acta Metall. 24 (1976) 333.

[23] Launay, C., Bulou, A. and Nouet, J., Solid State Commun. 69 (1987) 539.

[24] Bruinsma, R. and Zangwill, A., J. Physique 47 (1986) 2055.

[25] Magee, C. L., Phase Transformation, Séminaire de l'A.S.M. (Chapman and Hall, London) 1970, p. 115.

[26] Guénin, G., Rios Jara, D., Morin, M., Delaey, L., PynN, R. and GoBIN, P. F., J. Physique Colloq. 43 (1982) C4-597.

[27] Cochran, W., Adv. Phys. 9 (1960) 387.

[28] Coffey, C. S., Phys. Rev. B (1986). 